Int. J. Dev. Biol. 64: 227-237 (2020)

https://doi.org/10.1387/ijdb.190120dm

\title{
Homeobox genes in endometrium: from development to decidualization
}

\author{
NANCY ASHARY, SANIYA LAHERI and DEEPAK MODI* \\ Molecular and Cellular Biology Laboratory, ICMR-National Institute for Research in Reproductive Health, \\ Indian Council of Medical Research (ICMR), Parel, Mumbai, India
}

\begin{abstract}
The eutherian species evolved an elaborate uterus to allow viviparity. For successful pregnancy, the uterus must not only be differentiated, but must also function optimally and any defects in uterus differentiation and/or function can lead to infertility. The homoebox gene HOXA10 has emerged to be a key player in both uterine development and its optimal functioning in adulthood. Within the $A b d-B$ family, the posterior Hoxa genes play a dominant role in anterioposterior segmentation of the Müllerian ducts in mammals, with Hoxa10 having a central role in uterine segmentation. In the adult endometrium, HOXA10 is expressed by endometrial cells and is regulated in a cyclic manner under the influence of ovarian steroids. During embryo implantation, expression of HOXA10 is increased in endometrial stromal cells by signals from the embryo to govern stromal cell transformation to decidual cells. Once decidualization is initiated, HOXA10 is rapidly downregulated to activate expression of pro-invasive factors to promote trophoblast invasion. We propose that HOXA10 governs embryo implantation in a three-step process: 1) acquisition of endometrial receptivity, 2) responding to signals from the blastocyst to modify receptive endometrium for decidualization 3) making the decidua conducive for trophoblast invasion and placentation. There is currently ample evidence that expression of HOXA10 is deregulated in a variety of "endometriopathies" such as endometriosis and endometrial cancers. Overall, HOXA10 appears to be the master regulator of endometrial health and a central determinant of fertility in mammals.
\end{abstract}

KEY WORDS: HOXA10, HOX, Müllerian duct, implantation, trophoblast, invasion, pregnancy, fertility, infertility

\section{Introduction}

Oviparity (egg laying) to viviparity (live birth) is one of the most fundamental transition during course of vertebrate evolution that requires changes in female reproduction anatomy and physiology. Although viviparity has evolved independently in multiple lineages of fishes, amphibians and reptiles, except for the eutherian mammals other species do not have all the steps of pregnancy. By definition, the key features of the initiation of pregnancy include the implantation of a highly invasive blastocyst; 'acceptance' by the mother, and creation of a feto-maternal unit (placenta). These processes are unique to the eutherian mammals and do not occur in other viviparous species. To the best of our knowledge, such integrated form of pregnancy is limited to eutherian (aka 'placental') mammals and that requires development of specialized organ structures in the female reproductive tract.

In most species the female reproductive tract is a hollow tube that includes an anterior ciliated funnel to capture eggs released from the ovary and a posterior muscular tube that aid in egg provisioning, shell deposition, egg storage and ends in the vagina. However, in the eutherian species, this tube underwent extensive modifications during evolution to develop anatomically and functionally distinct structures termed as the oviduct/fallopian tube, the uterus and the cervix. About 105 million years ago, placental mammals evolved with an elaborate uterus that allowed implantation of the hatched embryos, promoted invasion by the placenta and aided in maternal tolerance of the fetus for long gestation periods. The evolutionary, developmental and molecular aspects of embryo implantation and placentation has been a subject of recent review (Aplin and Ruane, 2017; Chavan et al., 2017; Ashary et al., 2018). Herein we review on how a developmental factor HomeoboxA10 (HOXA10), which is

Abbreviations used in this paper: $\mathrm{AMH}$, anti-Müllerian hormone; A-P, anteroposterior; HOXA10, homeoboxA10; shRNA, small hairpin RNA.

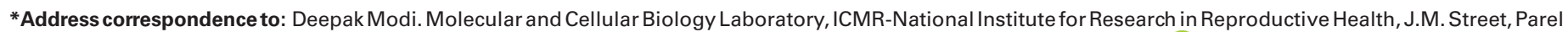

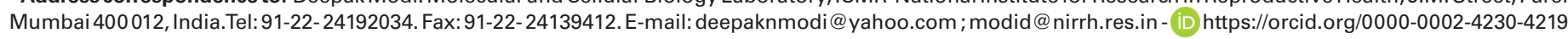

Supplementary Material (one table) for this paper is available at: https://doi.org/10.1387/ijdb.190120dm

Submitted: 25 June, 2019 Accepted: 25 July, 2019.

ISSN: Online 1696-3547, Print 0214-6282

(C) 2020 UPV/EHU Press

Printed in Spain 
required for segmentation and specification of the uterus, governs its adult functions pertaining to endowing endometrial receptivity, embryo implantation, placentation and regulating maternal immune tolerance for a successful pregnancy.

\section{Homeobox genes}

The homeobox genes are classical developmental factors involved in the body segmentation. These genes contain a conserved DNA sequence called homeobox that encodes the homeodomain. Homeodomain are helix-loop-helix-turn-helix structure which is responsible for recognizing and binding specific DNA sequences to regulate expression of target genes. In mice and humans, there are at least $39 \mathrm{HOX}$ genes arranged in 4 clusters and designated as $H O X A, H O X B, H O X C$ and HOXD. Unlike the Drosophila and other organisms, the HOX gene clusters in most mammals generally show a considerable overlap in their expression profiles suggestive of their functional redundancy (Parker et al., 2016). However,
A Wild type

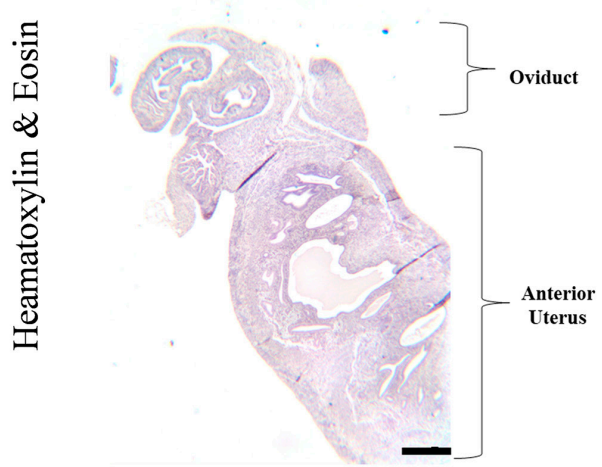

B

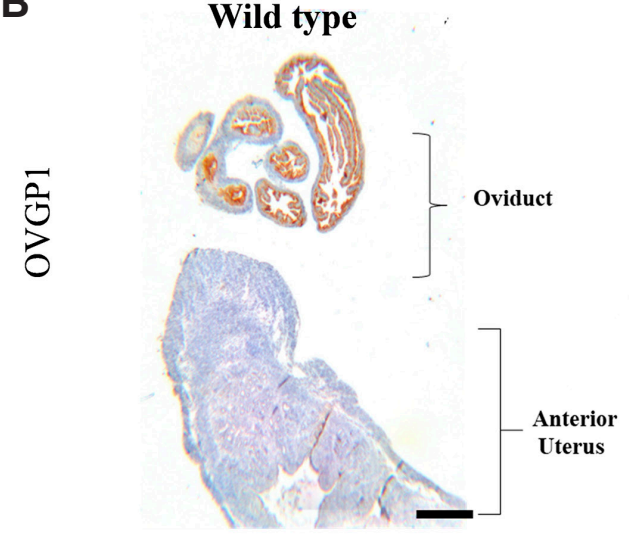

C

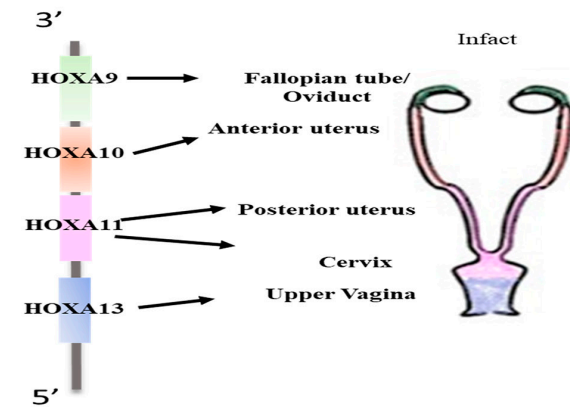

\section{HOXA10 Hypomorphs}

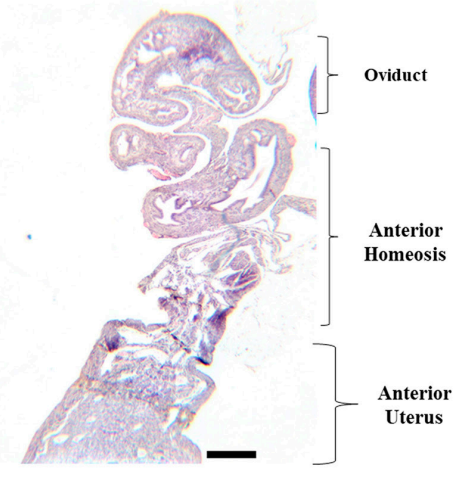

HOXA10 Hypomorphs

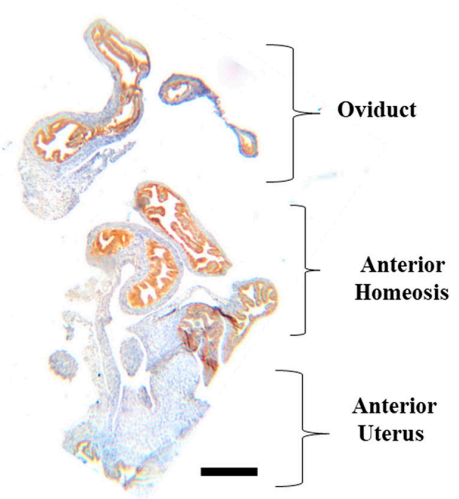

despite these overlapping patterns of expression the HOX genes have a well-defined role in anteroposterior (A-P) body axis plans specifically in regulating segmental patterns of hindbrain, skeleton axis and the limb axis (Parker et al., 2016).

\section{HOX code of Müllerian duct segmentation}

During the sexually indifferent stages, the urogenital system i.e. the kidneys and the reproductive tracts develop from the mesonephric and paramesonephric ducts. The paramesonephric ducts are the origin of the female reproductive tract that arises from longitudinal ridges of the peritoneal lining of the coelom. In all species including humans, in the $X Y$ embryos the Wolffian ducts regress by the production of the anti-müllerian hormone $(A M H)$, while gonads of the $\mathrm{XX}$ embryos produce minimal amounts of $\mathrm{AMH}$ resulting in their retention (Modi et al., 2006).

Postnatally, the müllerian ducts have to undergo A-P patterning such that the anterior segment develops in to an oviduct, the middle segment develops as the uterus, the posterior segment develops in to a cervix and the distal most portion develop in to upper vagina. This segmentation of the müllerian duct is governed primarily by the posteriorlyexpressed Drosophila gene abdominal-B (Abd-B) homeobox cluster (Hox). There are 16 genes in the posterior Abd-B cluster of which the HOXA genes play a significant role in segmentation of the müllerian duct. Based on expression patterns of the HOX genes and the data on mice knocked out for individual $\mathrm{HOX}$ genes have revealed the HOX code of müllerian duct differentiation (Fig. 1). In the mice, prior to birth, Hoxa9, Hoxa10, Hoxa11, and Hoxa13 are detected along the length of the müllerian duct, however two weeks postnatally, a spatial Hox axis is established following the paradigm of spatial collinearity. Hoxa9 is detected in the differentiating oviduct, both Hoxa10 and Hoxa11 in the uterus, Hoxa11 in the uterine cervix and Hoxa13 in the upper vagina (Taylor et al., 1997).

Fig. 1. HOXA10 is required for uterine specification in mouse Mullerian ducts. Mice hypormphic for HOXA10 were generated by overexpressing small hairpin RNA (shRNA) using testicular transgenesis. Three animals of the F2 generations showed anterior homeosis. (A) Histological analysis (Heamatoxylin \& Eosin staining) of the uteri of wild type and HOXA10 Hypomorphs. Scale bar in is $250 \mu \mathrm{M}$. (B) Localization of OVGP1 protein in the uteri of wild type and HOXA10 Hypomorph. Paraffin sections probed with anti OVGP1 antibody as described earlier (Laheri et al., 2017). Brown staining is indicative of positive reaction. Blue is counterstain of the nuclei with haematoxylin. Scale bar in is $250 \mu \mathrm{M}$. (C) HOX code of the developing Mullerian duct system and effect of loss of HOXA10 leading to anterior homeosis. Genes specifying different tissues of the female reproductive tract in infant and adult mice. 
A

Fig. 2. Collinearity of the posterior HOX cluster in the adult human female reproductive tract. (A) Structural organization of the mammalian HOX cluster. The AbdB cluster is shown in red. (B) mRNA levels of posterior HOX genes in different segments of human female reproductive tract. The genes are listed in rows and the tissues in the columns. The mRNA levels (RPKM) values for each gene was retrieved from the Human Protein Atlas database (https://www.proteinatlas. org). The intensity of thee colour in each block depicts the transcript abundance. Blocks which do not have a colour have RPKM values below 0.1. (C) Persistence of the HOXA code of the adult human female reproductive tract.

C
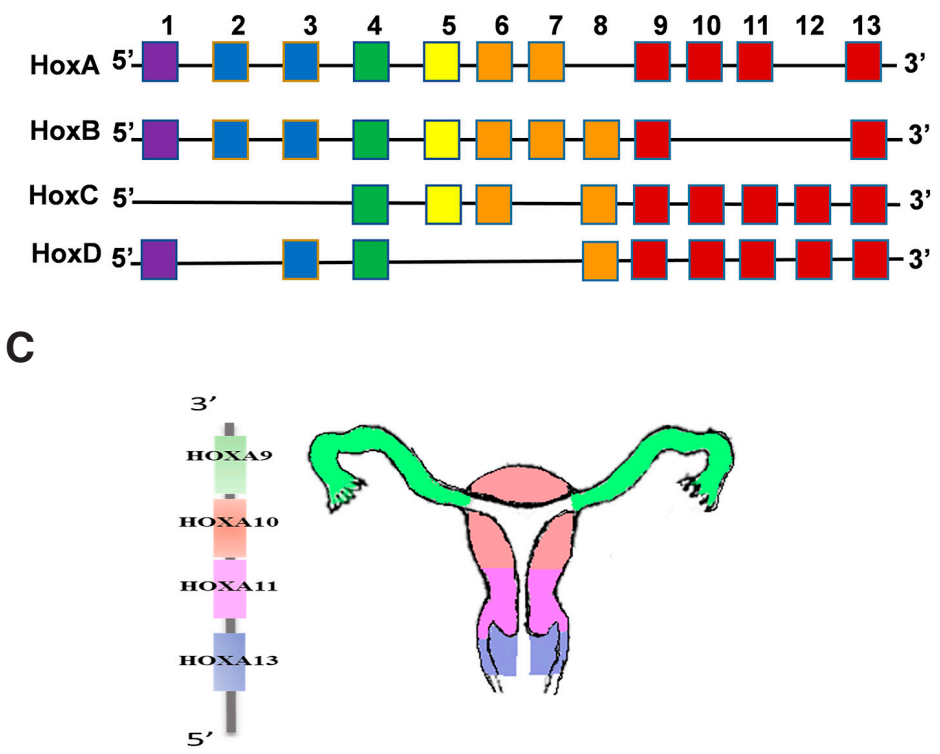

B

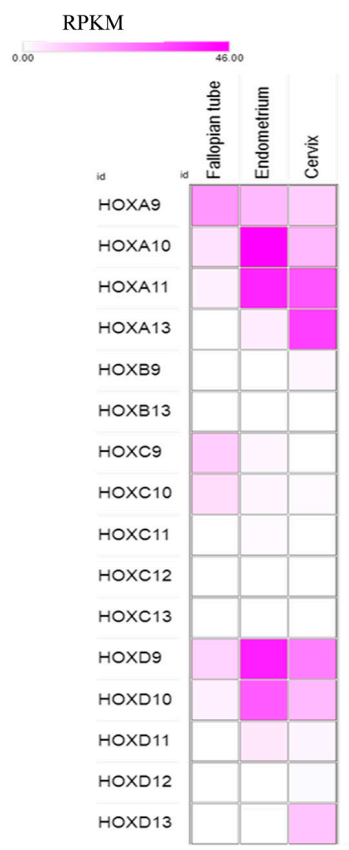

The critical roles of the Hox genes in segmentation are evidenced by targeted mutagenesis of these genes, which leads to regionspecific homeotic transformations. Hoxa10 is central to segmentation of the female reproductive tract specifically to demarcate the boundary of the fallopian tube and the anterior uterus is evident from studies in the Hoxa10 knockout mice (Satokata et al., 1995). In these animals, the müllerian duct is specified correctly, but the anterior portion of the duct has highly coiled structures resembling the oviduct. This phenotype has been faithfully recapitulated in our laboratory using mice hypomorphic for Hoxa10 (Fig. 1). We have developed mice that transgenecially expressed shRNA against Hoxa10 resulting in animals that have reduced expression of the HOXA10 protein. In a proportion of these animals, the anterior portion of the tube appeared thinner and at times coiled. Histologically, Hoxa10hypomorphs had multiple segments of the coiled structures in the anterior uterus that resembled the oviduct (Fig. 1). Furthermore, these extended coiled structures abundantly expressed the oviduct specific marker OVGP1 (Fig. 1) indicating that the loss of HOXA10 not only leads to a structural homeotic transformation but they also carry the molecular features of a transformed segment. These results imply that HOXA10 is essential for segmentation of the anterior müllerian duct in the oviduct and the uterus, the other HOX orthologous/paralogues cannot compensate for its loss.

HOX genes were once considered to be expressed only during embryonic development, however, in the female reproductive tract the persistence of HOX gene expression has been well characterized. Consistent with the developmental pattern, the posterior HOX genes are segmentally expressed in the adult female reproductive tract. (Fig. 2). Quantitatively, the HOXA cluster is most abundantly expressed in the female reproductive tract, HOXB9 is weakly expressed in the cervix, most members of $H O X C$ cluster are not expressed in the female reproductive tract, and the HOXD cluster is expressed majorly in the endometrium (Fig 2). Amongst the three clusters, the HOXA cluster genes are expressed segmentally and faithfully recapitulate the developmental pattern. Transcripts for HOXA9 are highest in the fallopian tube; HOXA10 and HOXA11 are maximally expressed in the endometrium; HOXA11 is expressed in endometrium and cervix and HOXA13 is highly expressed in the cervix. Interestingly, the HOXC family does not show any such consistency, with only $\mathrm{HOXC9}$ and $\mathrm{HOXC10}$ are being weakly detected in fallopian tube. However, parallel to HOXA cluster, the segmental expression of HOXD cluster is relatively maintained, although lesser in abundance as compared to HOXAgenes. HOXD9 is detected in fallopian tubes, endometrium and cervix, HOXD10 in the endometrium and cervix, HOXD11 only in the endometrium while HOXD13is only detected in cervix. These observations imply that, while in the flies and most organisms, the HOX cluster is developmentally switched off, in the mammalian female reproductive tract the segmental expression of these genes occurs relatively later and is persistent well until adulthood. The functional significance of retaining the developmental segmentation pattern of the HOX cluster is yet not clear; it is possible that the persistent HOX gene expression may be a mechanism to retain developmental plasticity. Interestingly, ectopic overexpression of the individual genes of HOXA cluster in ovarian surface epithelium can induce müllerian duct like phenotypes. Overexpression of Hoxa9 leads to papillary tumors resembling the oviduct, overexpression of Hoxa10 and Hoxa11 induced morphogenesis of endometrioid-like structures in the ovarian surface epithelium (Cheng et al., 2005). Preliminary data from our laboratory has shown that knocking down HOXA10 from the endometrial epithelial cells, results in gain of expression of markers associated with oviduct epithelium, suggesting that the persistence of HOX genes in the adults may be required to maintain the developmental phenotypes. Amongst the HOX genes, HOXA10 has been well characterized for its expression in the adult endometrium. HOXA10 is a sequence-specific transcription factor that binds to the DNA sequence 5-AA[AT]TTTTATTAC-3 on 5' regulatory region of genes to regulate their transcription ( $\mathrm{Du}$ and Taylor, 2015). The human HOXA10 gene encodes for a 410 amino acid protein with an approximate molecular mass of $42 \mathrm{kDa}$ with a DNA binding domain between amino acids 336-395. Uniprot associates $\mathrm{HOXA10}$ with DNA binding transcription factor and histone 
deacetylase activity as its major molecular function and anterior/ posterior segment specification as the major biological processes. Human HOXA10 has multiple phosphorylation, methylation, acetylation and ubiquitination sites. Recently, HOXA10 is also reported to undergo sumoylation (Jiang et al., 2017; Zhu et al., 2013).

HOXA10 protein is detected in the nucleus and cytoplasm of the epithelial and stromal cells of mouse, baboon, bonnet monkeys and human endometrium (Godbole et al., 2007; Godbole et al., 2017; Modi and Godbole, 2009). The mammalian endometrium is divided into two layers viz the stratum functionalis (the upper $2 / 3$ of the uterus from the lumen) and stratum basalis (the lower $1 / 3$ towards the myometrium). In the bonnet monkey endometrium, HOXA10 is more strongly expressed in the endometrial functionalis as compared to the basalis zone. HOXA10 is also detected in the myometrium (Godbole et al., 2007). While the functional significance of such differential distribution of HOXA10 in the two zones of the endometrium is unknown, it is speculated that such zonal distribution might endow specialized roles to the functionalis zone for implantation or menstruation. Since the HOX family of genes aid in the differential distribution of the morphogens (Parker et al., 2016), it is possible that zone-specific distribution of HOXA10 may aid the creation of boundaries of microenvironments within the uterus in preparation for implantation.

\section{Endometrial receptivity}

The endometrium undergoes cyclic changes under the influence of steroid hormones and is generally refractory to embryo implantation. Approximately day 21-24 of a 28-day human menstrual cycle (8-10 days posts ovulation), the uterus becomes "receptive" and enables blastocyst implantation. This phase is termed as "window of receptivity" or "window of implantation", the endocrine regulation of the menstrual cycle and the role of hormones in endowing receptivity to the endometrium has been a subject of extensive review (Evans et al., 2016). Several transcription factors, integrins and their ligands, cytokines and growth factors are differentially expressed during the cycle and many of these are steroid hormone regulated; a specific signature of the receptive endometrium has been identified (Altmäe et al., 2017), The genetic and proteomic networks that endow this receptive phase are also being dissected (Bajpai et al., 2012; Bhusane et al., 2016; Padmanabhan and Laloraya, 2016).

HOXA10 seems to be one of the players that endow receptivity to the endometrium. The expression of HOXA10 and HOXA11 varies in a cycle-dependent manner. In the mouse, monkeys and human endometrium, HOXA10 is weakly expressed in the proliferative phase, the expression is robustly increased in the secretory phase (Modi and Godbole, 2009; Taylor et al., 1998). Irrespective of the species, the expression of HOXA10 peaks in endometrial epithelial and stromal cells during the window of receptivity (Akbas et al., 2004; Godbole et al., 2007; Gui et al., 1999; Xu et al., 2014). The cyclic variations in the expression of HOXA10 in the endometrium are regulated by the sex steroids estrogen and progesterone. Evidence for estradiol-mediated regulation of HOXA10 expression came from in vitro observations where a significant increase in HOXA10 mRNA was observed in human endometrial epithelial and stromal cells treated with estradiol (Akbas et al., 2004; Taylor et al., 1998). The 5' regulatory region of the human HOXA10 contain estrogen response elements (EREs) that bind both estrogen receptor alpha
(ERa) and beta (ER $\beta$ ) to increase HOXA10 transcription (Akbas et al., 2004). While this estradiol mediated increase in HOXA10 is essential for fertility, the same mechanism compromises the fertility in the context of xenoestrogens. Xenoestrogens are estrogen like substances that can bind to ERs and imitate their effects. Humans are exposed to a wide variety of Xenoestrogens that have long lasting effects by affecting DNA methylation. The studies have shown that xenosestrogens like diethylstilbestrol, methoxychlor and bisphenol A alters HOXA10 expression by altering its methylation leading to developmental anomalies in the female reproductive tract resulting in permanent alteration of gene in the adult (Du and Taylor, 2015). It is possible that the xenoestrogen mediated effects on the HOXA10 gene might be a cause of infertility in a number of cases. Indeed, altered HOXA10 expression and changes in its methylation are observed in a number of "endometriopathies" (see discussion below and supplementary Table 1).

Akin to estradiol, HOXA10 is also regulated by progesterone. In primary endometrial stromal cells and epithelial cells, progesterone alone increases the expression of HOXA10; combined estradiol and progesterone treatment that mimic the receptive state of the endometrium having a synergistic effects (Gui et al., 1999; Modi and Godbole, 2009; Taylor et al., 1998). While, the 5' regulatory region of $H O X A 10$ has progesterone response elements, whether these are functional and bind to progesterone receptor to regulate HOXA10 is yet not known. Nevertheless, that progesterone is essential for maintenance of HOXA10 expression in the receptive endometrium is evident from the studies in mouse, human and monkeys where treatment with progesterone receptor modulators down regulate its expression in the mid luteal phase (Godbole et al., 2007; Whitaker et al., 2017) indicating that HOXA10 may be involved in endometrial receptivity.

The notion that HOXA10 is required for endometrial receptivity came from studies in Hoxa10 knockout mice (Hoxa10-/-). Altered expression of several receptivity related genes and pinopodes formation (the morphological hall mark of receptive endometrium) are observed in endometrium of Hoxa10-/- mice (Bagot et al., 2001; Lim et al., 1999), underscoring the requirement of HOXA10 in endowing receptivity in the rodents. That HOXA10 is required for endometrial receptivity in primates came from our studies in bonnet monkeys that were rendered infertile by low dose of antiprogestins which did not affect the hormone profiles but the uterus was non-receptive (Patil et al., 2005). As compared to luteal phase receptive controls, in the non-receptive endometrium, the expression of HOXA10 was down-regulated in the luminal and glandular epithelial cells along with the stroma in the functionalis zone but not in the basalis region (Godbole et al., 2007). These observations together suggest that HOXA10 is essential for endowing receptivity to the endometrium and its loss may cause implantation failure. Indeed, mice knockout for Hoxa10 suffer from uterine factor infertility as the females produce normal number of embryos, but they fail to implant (Benson et al., 1996; Satokata et al., 1995) indicating that HOXA10 is indispensable for endowing receptivity in the endometrium.

\section{Embryo implantation}

It has long been assumed that a receptive endometrium is a passive tissue and gain of "receptive state" is sufficient for embryo implantation. However, studies from our lab and others have shown 
that there are several morphological and molecular changes in the receptive endometrium in presence of an embryo. Using bonnet monkeys as our study model, we demonstrated that in the presence of the embryo there are distinct morphological changes in the luminal epithelium, glandular epithelium and stroma of the endometrium even prior to implantation. As compared to receptive control, in the monkeys that were mated (and the presence of embryo was verified) there was extensive proliferation, loss of columnar shape of luminal epithelial cells with diffusion of apico-lateral gap junction of the endometrium epithelial cells was observed (Rosario et al., 2005a;2008). Asimilar loss of junctional protein E cadherin and cell proliferation is observed in the mouse endometrium at the site of embryo implantation (Ashary et al., 2018). Beyond the epithelium, in presence of an embryo, the stromal cells undergo extensive compaction, there is loss of edema and the cells express markers associated with decidualization (Rosario et al., 2005a). In addition, there is increased angiogenesis in the endometrium in response to embryonic signals (Rosario et al., 2005a). These changes occur in the endometrium due to secretions from the embryo as similar morphological changes are seen in the endometria of baboons infused with embryonic factors human chorionic gonadotropin (hCG) and Interleukin 1beta (IL-1 $\beta$ ) (Modi et al., 2012; Modi and Bhartiya, 2015).

The morphological changes in the endometrium are also paralleled by several molecular changes that include increased expression of estrogen receptor alpha (ER alpha), transforming growth factor beta (TGF $\beta$ 2), Interleukin 6 (IL-6), Glycodelin and Integrin's in the endometrium of bonnet monkeys in presence of an embryo (Nimbkar-Joshi et al., 2012; Rosario et al; 2005 b,c; Rosario et al., 2008). We also discovered a novel isoform of protein kinase a regulatory subunit which is transcribed at higher levels in endometria of monkeys in presence of an embryo (Rosario et al., 2009). Interestingly, comparable molecular changes are also observed in endometria of baboons infused with embryonic factors (human CG and IL1b) and these are summarized by us previously (Modi et al., 2012). That embryonic factors can also induce molecular changes in the humans came from analysis of endometrial biopsies derived from women infused with hCG. As compared to luteal phase biopsy, the tissue obtained after hCG infusion had higher expression of estrogen receptor alpha 1 (ESR1), progestrone receptor (PGR) and $\alpha$-smooth muscle actin ( $\alpha$-SMA) which are also observed in monkey endometrium in presence of an embryo (Strug et al., 2016) These observations suggest that the embryo superimposes the molecular signature of a receptive endometrium prior to implantation.

To dissect the molecular features of embryo-endometrial crosstalk, we focused our studies towards the changes that occur in the luminal epithelium in the receptive endometrium during the course of implantation While HOXA10 was thought to be essential for receptivity and its expression is high in the luminal epithelial cells in the mid luteal receptive stage endometrium, to our surprise we observed loss of HOXA10 exclusively in the luminal epithelium of bonnet monkey in the presence of embryo (Godbole et al., 2007). Loss of HOXA10 expression is also observed in mouse endometrial luminal epithelium during the course of embryo implantation (our unpublished data, Satokata et al., 1995). The functional consequence of the loss of HOXA10 in the endometrial epithelium in the context of embryo implantation is still being explored.

Beyond HOXA10, a recent study from our lab has shown that oviductal glycoprotein 1 (OVGP1) is expressed in luminal epithelium of endometrium only in presence of an embryo. OVGP1 is a $120 \mathrm{kDa}$ secretory glycoprotein that is synthesized and secreted by the epithelial cells of the mouse oviduct, ovary, testis and epididymis, but not by the endometrium (Laheri et al., 2017). However, we serendipitously discovered that there is expression of OVGP1 exclusively in the luminal epithelium when embryo initiates implantation in the receptive stage mouse endometrium, suggesting that OVGP1 should be induced by embryonic factors (Laheri et al., 2018). Indeed, we observed increased expression of OVGP1 in human endometrial epithelial cell line (Ishikawa cells) by the ovarian hormone estrogen and human CG (Laheri et al., 2018). In vivo, along with progesterone, there is a transient estrogen surge which is necessary for embryo implantation to occur. We observed that the combined effect of estrogen, progesterone and hCG profoundly increased OVGP1 mRNA expression (Laheri et al., 2018). Interestingly, knocking down HOXA10 also increases OVGP1 in endometrial epithelial cells (unpublished). Thus, OVGP1 is specifically induced in the luminal epithelial cells, most likely in response to steroid hormones and loss of HOXA10 in combination with the embryonic signals; it must have a role in implantation. Indeed, knocking down of OVGP1 in human endometrial epithelial cell line altered the expression of several implantation related factors including cytokines like leukemia inhibitory factor (LIF) and IL6, there is also reduced adhesive ability of trophoblast cells in response to media derived from OVGP1 depleted epithelial cells (Laheri et al., 2018). While these results are early and has obvious caveats, it is tempting to propose that the embryo driven signaling leads to downregulation of HOXA10, followed by increase in OVGP1 production in the endometrial epithelium is required to create a milieu that is conducive towards implantation.

With the accumulating data, it is getting increasingly clear that remarkable changes occur in the structural and molecular profile of endometrium in response to the embryo and these changes are highly specific and localized in nature. However, the functional implications of such observations remain far from clear. This is mainly due to our inability to perform genetic manipulations specifically in the endometrium before implantation, after the gain of receptivity. However, in vitro studies have tried to decipher the functional connotations of embryo to endometrial discussion during implantation. While it would be beyond the scope of this review to discuss these studies, it appears embryo-endometrial cross-talk permits the endometrium with a bio-sensing ability to judge the embryo quality.

In recent years, experimental evidence haves emerged suggesting that the endometrium has an ability to discriminate between normal and abnormal blastocysts. Incubation of morphologically normal and abnormal human embryos induces distinctly differential molecular changes in endometrial stromal cells in vitro. Soluble signals from the developmentally incompetent human embryos induce an endoplasmic reticulum stress response that leads to the termination of receptivity, ultimately leading to menstruation; the signals derived from competent embryos evoke a response that is supportive towards implantation (Macklon and Brosens, 2014). These results imply that the embryo-endometrial cross-talk enable the mother to judge the embryo quality during implantation. These effects seem to be due to differential secretions of selected microRNAs and/or the microvesicles by implantation competent and implantation incompetent blastocysts. The embryo derived miRNA 
and the microvesicle cargo that aid in the process of implantation is being identified and has been reviewed by us recently (Kurian and Modi, 2018).

Based on the above studies, we propose a two phase response of the endometrium to govern implantation. The first involves attainment of "receptivity status" in the endometrium; the second phase involves responses of the receptive endometrium towards the incoming embryo which allows it to attain a "selector status" and judge the developmental competence of the implanting embryo. Based on the blastocyst competence the endometrium decides to either continue with implantation and further decidualization or abort the receptive status to culminate in to menstruation. What are the endometrial tools that empower it to have such bio-sensing ability are hitherto unrecognized.

\section{HOXA10 in stromal cell decidualization}

Once the endometrium attains the receptivity state it undergoes decidualization: A postovulatory endometrial remodeling process which occur in preparation for pregnancy. During decidualization there is secretory transformation of the uterine stroma, influx of specialized uterine natural killer cells, and vascular remodeling (Evans et al., 2016; Gellersen and Brosens, 2014). In its strictest sense, decidualization is the morphological and biochemical reprogramming of the endometrial stromal compartment into highly specialized cells with distinctive functions. Decidualization only occurs in species where placentation involves breaching of the luminal epithelium by the trophoblast (Gellersen and Brosens, 2014). In humans decidualization is obligatory and dependent on ovarian steroids; in almost all other species including non-human primates, the presence of an embryo or a physical assault to the endometrial epithelium is essential for decidualization (Evans et al., 2016). In humans around day 23 of the 28-day menstrual cycle, decidual transformation is first apparent in the stromal cells surrounding the spiral arteries and is referred to as the pre-decidual response, this response is flared-up in case the embryo successfully implants. In most other species, decidualization occurs only upon embryo implantation.

Decidualization involves large scale reprogramming of the stromal cells to achieve the decidual phenotype which is marked by secretion of a variety of proteins amongst which prolactin (PRL) and insulin-like growth factor binding protein-1 (IGFBP-1) are the principle ones (Okada et al., 2018). This process of endometrial stromal cell decidualization can be faithfully recapitulated in vitro both at morphological and molecular level by treating primary cultures of human endometrial stromal cells with estrogen and progesterone which can be hastened by addition of cAMP (Lucas et al., 2018.).

As discussed above, in presence of an embryo, the expression of HOXA10 is increased in the endometrial stromal cells when they initiate decidualization. This increase in expression of HOXA10 is also observed in the endometrial stromal cells during the course of in vitro decidualization using estrogen and progesterone alone (Godbole and Modi, 2010) and also in combination with cyclic adenosine monophosphate (cAMP) (Lu et al., 2008). Decidualization of stromal cell requires HOXA10 is evident from the fact that knocking down HOXA10 in the human endometrial stromal cells inhibits the expression of decidual markers (Godbole and Modi, 2010); decidualization is also compromised in endometria of mice knockout for Hoxa10 -/- (Lim et al., 1999). The molecular mechanism by which HOXA10 regulates endometrial stromal cell decidualization has been worked out. It is known that the endometrial stromal cells undergo proliferation just prior to decidualization and HOXA10 seem to be controlling this process. In the endometria of mice knockout for Hoxa10, there is inhibition of stromal cell proliferation mediated by progesterone (Lim et al., 1999; Yao et al., 2003) and this phenotype can be rescued by overexpression of cell cycle genes Ccnd3 and FoxM1 (Gao et al., 2015). Even in humans, inhibition of HOXA10 during the course of decidualization impedes cell proliferation by affecting the expression of cell cycle genes (Lu et al., 2008). Thus, proliferation seems to be the rate limiting step in stromal cell decidualization and HOXA10 seems to be key regulator.

\section{Decidualization is multiphasic in nature}

The observations that HOXA10 is highest during the window of receptivity and its loss impedes decidualization led to the notion that HOXA10 must be sacrosanct for differentiation of decidua. However, we had observed that the during the course of in vitro decidualization there were multiple waves of HOXA10 expression (Godbole and Modi, 2010). During a 24 day course of in vitro decidualization of human endometrial stromal cells by steroid hormones, there was an initial increase in expression of HOXA10 mRNA by day 8 which drops subsequently only to rise again by day 24. We further observed that while the initial rise of HOXA10 (on day 8) was essential for endometrial stromal cell decidualization as the expression of decidual markers dropped significantly, loss of HOXA10 at the later time points (day 24) surprisingly increased the expression of decidual markers (Godbole and Modi, 2010). A similar observation was also reported (Kim et al., 2007) where the loss of HOXA10 increased the expression of the decidual Insulinlike growth factor-binding protein 1 (IGFBP1) in cultured decidual cells. These observations led us to propose that while HOXA10 may be essential for initial decidual transformation of endometrial stromal cells, it must be down regulated for their terminal differentiation. These findings gained significance when we compared the expression profiles of HOXA10 in the endometrium and decidua in vivo. We observed that in the decidua of human and baboons (obtained at 10 weeks and 60 days of pregnancy respectively) the expression of HOXA10 is far lower than that observed in the stromal compartment of endometrium of implantation stages (Godbole et al., 2017). Global gene profiling revealed that loss of HOXA10 in the decidualized endometrial stromal cells promoted the expression of several cytokines and growth factors associated with the decidua (Godbole et al., 2017). These observations prompted us to postulate that endometrial decidualization is perhaps a multiphasic process, where there is initial transformation of stromal to decidual cells require HOXA10; the next phase involves downregulation of HOXA10 leading to its further differentiation (Fig. 3). Indeed, such multiphasic decidual response has been also observed in time dependent transcriptome profiling of in vitro decidualizing human endometrial stromal cells and is associated with the similar rise and fall of HOXA10 (Rytkönen et al., 2018). Single cell RNAseq of first trimester human decidua or in vitro decidualized cells have identified 2-3 sub-populations of stromal cells that have unique transcriptome signature (Suryawanshi et al., 2018; Vento-Tormo et al., 2018). Presently, we do not know if HOXA10 is differentially 
Fig 3. Diphasic role of HOXA10 in the endometrium during embryo implantation. Initially at the receptive stage, the increase in HOXA10 expression in stromal cells in response to embryonic stimuli induces proliferation and initial transformation. In the second step, in transformed stromal cells, downregulation of HOXA10 promotes decidualization and cytokine production. Together, these events aid trophoblast invasion and embryo implantation (See Fig. 4 for the mechanism).

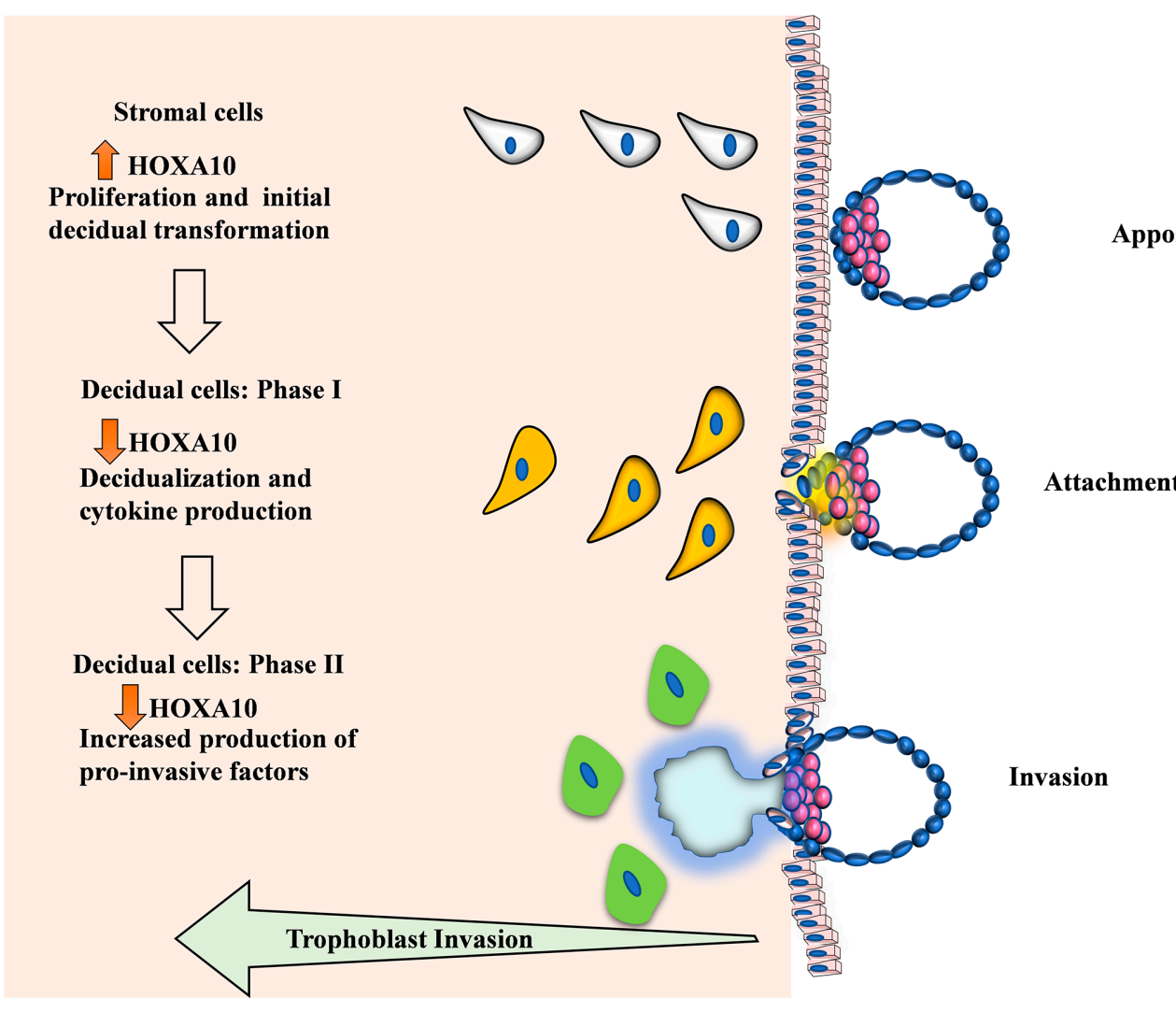

expressed in these decidual cell types, but based on our data we suspect that the oscillating kinetics of HOXA10 in the decidual cells that may regulate this process of multiphasic decidualization.

\section{HOXA10 in decidua governs trophoblast invasion}

Once the endometrium encounters a developmentally competent blastocyst it implants in the endometrium and the trophoblast cells begins to invade in to the maternal decidua to establish placentation. Trophoblast cells inherently can invade any tissue; however, its invasion is highly controlled in the pregnant endometrium, thus leading to the notion that decidua must restrain trophoblast invasion (Sharma et al., 2016). However, studies in knockout mice for various genes have shown that decidualization is central for successful embryo implantation and placentation (Gellersen and Brosens, 2014). The notion that decidualization is required for trophoblast invasion came from our in vitro studies using supernatants derived from highly purified human endometrial stromal cells. We observed that supernatants derived from the in vitro decidualized cells (but not the primary stromal cells) increased the invasiveness of trophoblast cell lines JEG3 and ACH3P (Godbole et al., 2011). The invasion promoting ability of the decidual secretome is by its ability to activate matrix metalloprotease transcription and activity in the trophoblast cells (Godbole et al., 2011) that aids in matrix degradation. To understand how decidualization aids in trophoblast invasion we profiled the secretome of the decidual cells and observed that as compared to stromal cells, the supernatants from the in vitro decidualized cells have enhanced levels of several pro-invasive molecules such as cytokines and growth factors (Sharma et al.,
2016). Thus, decidualization driven changes in the endometrial stromal cells create a microenvironment favorable for implantation and trophoblast invasion (Sharma et al., 2016).

Interestingly, we further observed that while decidualization of endometrial stromal cells is essential for trophoblast invasion, this ability is further enhanced upon loss of HOXA10. Our in vivo studies in humans and baboons revealed that the levels of HOXA10 are very low at decidua basalis near placentation as compared to decidua parietalis away from placenta. This led us to speculate that loss of HOXA10 in the decidual cells might promote trophoblast invasion. Indeed, as compared to the stromal cells, the secretions from decidual cells enhanced trophoblast invasion, this ability was increased by several folds when HOXA10 was knocked down from the decidualized cells. The supernatants from HOXA10knock down decidua also activated matrix metalloproteinase (MMP) production in trophoblast cells (Godbole et al., 2017). We and others have earlier shown that during the course of invasion, the trophoblast cells actively switch their integrin profiles with initial requirements of the $\alpha v \beta 3$ that switch to $\alpha 5 \beta 1$ and $\alpha 6 \beta 1$ (Damsky et al., 1994; Mangale et al., 2008) . Our results showed that while the decidual supernatants by themselves marginally activates transcription of $\alpha 5$ and $\alpha 6$ subunits, the expression of av was not switched off (Godbole et al., 2011); however loss of HOXA10 in the decidual cells enhanced the expression of $\alpha 5$ and $\alpha 6$ subunits but downregulates ITGAVmRNA leading to a complete switch (our unpublished data). These results indicate that loss of HOXA10 in the deicidal cells, in a paracrine manner; alter trophoblast cell physiology towards a pro-invasive phenotype.

Global gene profiling and secretome analysis of the HOXA10 
knocked down decidual cells revealed increased expression and secretion of several cytokines mainly the gp130 cytokines Leukemia inhibitory factor (LIF), Interleukin -6 (IL6) and Interleukin -11 (IL11) that are major activators of trophoblast invasion (Dimitriadis et al., 2010; Godbole et al., 2017; Suman et al., 2012). We propose that the loss of HOXA10 in the decidual cells enhances the expression of pro-invasive factors to promote trophoblast invasion

We next asked what could be the mechanism by which loss of HOXA10 might regulate trophoblast invasion. Our earlier studies had shown that the gp130 cytokines activate STAT3 in the trophoblast cells to promote invasion (Suman et al., 2012) and STAT3 seems to be the converging point of all pro-invasive signaling in trophoblast cells (Sharma et al., 2016). These observations led us to speculate that the secretions derived from the HOXA10 depleted decidualized stromal cells must activate STAT3. As expected, we did observe increased STAT3 phosphorylation in the trophoblast cells treated with supernatants from HOXA10 depleted decidual cells as compared to supernatants from decidual cells having abundant HOXA10. This STAT3 activation is necessary and sufficient to drive invasion as blocking of STAT3 in the trophoblast cells impeded its invasion mediated by supernatants derived from HOXA10 depleted decidual cells (Godbole et al., 2017).

\section{Two-cell two transcription factor mechanism of trophoblast invasion}

The decidual control of trophoblast invasion seems to be a twostep process. The first involves the initial decidual transformation of the stromal cells under high HOXA10 making the tissue favorable for trophoblast invasion. In step 2, in the decidualized stromal cells, there is downregulation of HOXA10 leading to a burst in production of pro-invasive factors that in a paracrine manner activate STAT3 and stimulate MMP and alter integrin profile production in the trophoblast cells to potentiate their invasion (Fig. 4). In the decidual cells at the non-implantation sites, there would be sufficient
HOXA10 to limit the production of pro-invasive molecules there by restraining invasion. Thus, it is likely that during the course of placentation, in the decidual cells there could be oscillating kinetics controlled by HOXA10, both spatially and temporally creating localized gradients of pro-invasive factors that may differentially regulate STAT3 activity and MMP's production in the trophoblast cells leading to the fenestration of the developing placenta at the feto-maternal interface. We propose that decidual HOXA10 is like a rheostat that via the controlled production of pro-invasive factors keeps the STAT activity in the trophoblast in check to regulate invasion. Indeed, preliminary observations have suggested defective placental organization in the Hoxa10 hypomorphs (unpublished observations). It will be of interest to determine the HOXA10 activities in decidua of patients suffering from "placentopathies", which are the leading causes of many obstetric complications and maternal morbidity and mortality.

\section{HOXA10 and endometrial disorders}

As stated above Hoxa10-/-mice have malformation of uterus. In humans, mutations in HOXA10gene have been identified in women with müllerian anomalies like didelphic and bicornuate (Cheng et al., 2011; Ekici et al., 2013) but not in women with Mayer-Rokitansky-Kuster-Hauser syndrome, who lack the complete müllerian derivatives (Liatsikos et al., 2010). These observations underscore the importance of HOXA10 in uterine development in humans.

Several studies have reported that the expression of HOXA10 in endometria of women with uterine abnormalities including unexplained infertility and recurrent implantation failures (see supplementary Table 1 and references therein). Interestingly, irrespective of the pathology, the expression of HOXA10 seems to be downregulated in the abnormal endometrium. Furthermore, in women with endometriosis the expression of HOXA10 is lower in the eutopic endometrium. Mechanistically the downregulation of HOXA10 in most of these cases is attributed to hypermethylation

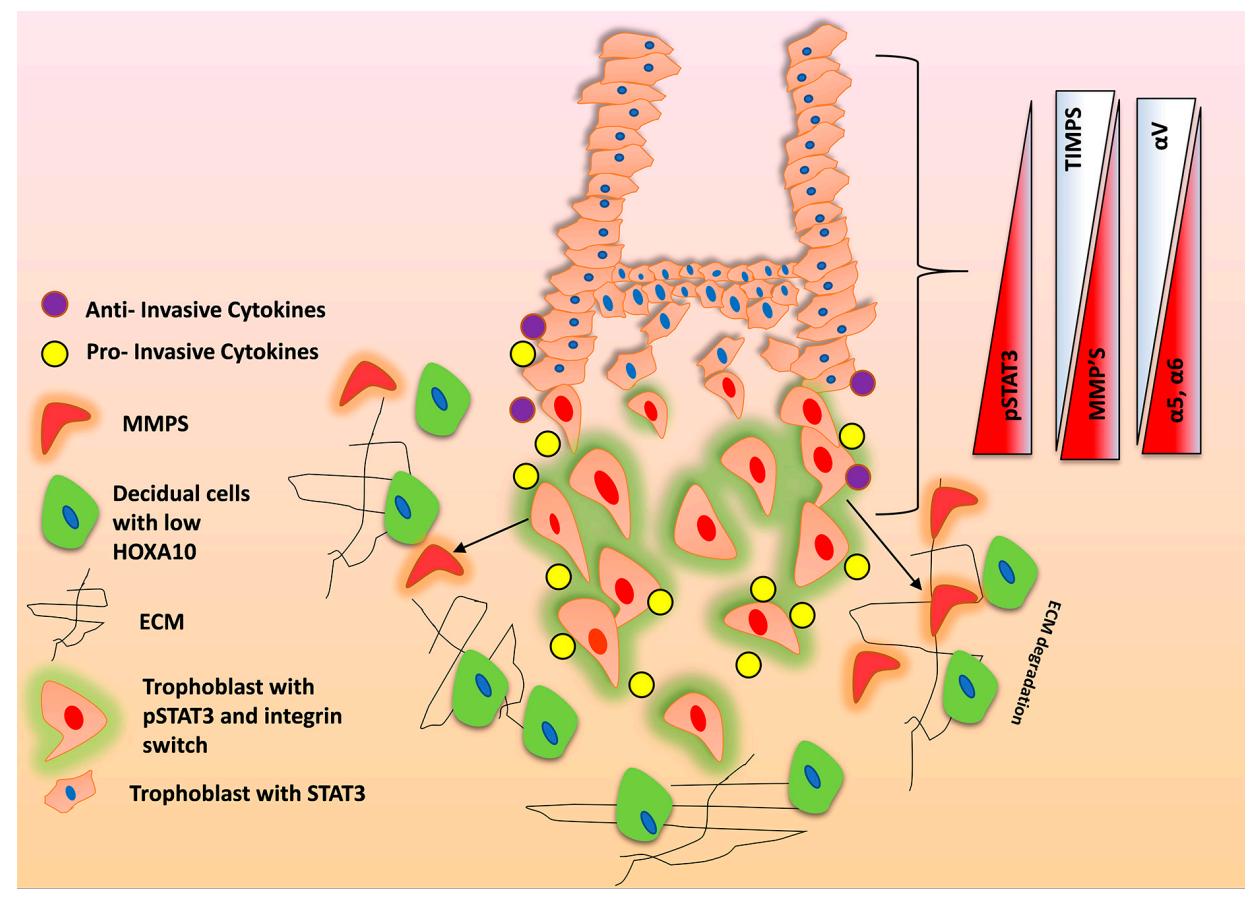

Fig 4. Mechanisms of decidual control of trophoblast invasion by HOXA10. Downregulation of HOXA10 increases levels of pro-invasive cytokines and represses anti-invasive cytokines. These pro-invasive cytokines (mainly LIF, IL6 and $(L-\beta)$ in a paracrine manner act on trophoblast cells to phosphorylate STAT3. This increases production of metalloproteinases (MMPS) and lead to integrin switching. This leads to extracellular matrix (ECM) degradation and increase in trophoblast invasion. 
of 5' regulatory region $\mathrm{HOXA10}$ gene (see supplementary table 1 for references). Based on these studies it has been postulated that infertility observed in these women may not be due to morphological defects but perhaps due to altered receptivity of the endometrium.

At present, it is unknown whether the loss of HOXA10 is cause or consequence of endometrial pathologies. Considering the requirement of HOXA10 in maintenance of normal endometrial functions, and that disruptions in HOXA10 expression due to xenoestrogens (during early life) affect uterine development, it is possible that loss of HOXA10 may actually cause some of these disorders (Du and Taylor, 2015). While experimental studies are lacking in these directions using Hoxa10 knockout mice, preliminary studies in our Hoxa10 hypomorphs, have shown phenotypes resembling endometrial hyperplasia in humans (our unpublished data). Interestingly, mice knockout for Hoxa10 regulated gene Fkbp52 have increased growth of endometriotic lesions and developed features resembling human endometriosis (Hirota et al., 2008). Together these results imply that loss of HOXA10 maybe a cause of atleast some of these endometrial disorders, more studies are needed to clarify the association of HOXA10 and endometrial pathologies and uterine disorders.

\section{Summary}

HOXA10 has emerged to be an extraordinary regulator of the uterus that allows the structural segmentation to specify the uterus during development and also function in the adult endometrium to regulate embryo implantation and early pregnancy. Developmentally, loss of HOXA10 leads to homeotic transformation in the müllerian ducts, in the adult endometrium HOXA10 functions to endow receptivity in the endometrium, govern embryo implantation and decidualization. Alterations in HOXA10 expression in the mouse and primates is associated with defects in uterine functions resulting in sterility suggesting that HOXA10 is perhaps the gatekeeper of endometrial health for fertility.

Our results suggest that in the context of HOXA10, embryo implantation is a three step process: 1 ) attainment of a receptive state in the endometrium; 2) modulation of the receptive endometrium in response to embryonic signals and finally 3 ) implantation of the embryo culminating in decidualization and placentation. From the current data it appears that unlike in the Drosophila where HOX genes have a role in segmentation, in mammals HOXA10 (and perhaps other genes of the HOX cluster) have acquired newer functions in the adult endometrium. In the adult female reproductive tract, these HOX genes (atleast HOXA10) behave like a signaling factor that regulate expression of many cytokines and growth factors to direct many cell non-autonomous functions (like placentation). To our knowledge, such activity has not been ascribed for HOX genes and leads us to postulate that HOXA10 is a molecular multitask protein that orchestrate a range of activities from uterine segmentation to embryo implantation. To fully appreciate the role of HOXA10, the need of the day is to identify and characterize the direct gene targets of this gene in development and adulthood. We believe that, increased understanding of how HOX transcription factors are integrated to regulate endometrial functions will provide us with an clear picture of physiological mechanisms involved in initiation of pregnancy and pathophysiological mechanism underlying the endometriopathies.

Clinically, the implications of such research are far reaching.
Currently, the treatment of recurrent miscarriages and implantation failure are highly empirical and ineffective. Endometriosis is a common disorder where we do not know the etiology and many women continue to suffer from it due to lack of specific and effective therapies. We believe that our newer understanding of the processes involved in uterine development to decidualization and placentation will aid in developing effective approaches to modulate implantation and treat pregnancy related complications. It is envisaged that such data will not only to assist in the development of specific therapeutics for fertility management but may also lead to the development of newer methods for endometrium based contraception.

\section{Acknowledgements}

We are thankful to Dr Geeta Godbole whose data has tremendously contributed towards our understanding of HOX genes in endometrium. We are thankful to Ms Anuradha Mishra for the data on Hoxa10 hypomorphs. We thank Mr Domdatt Singh for help in making the making for Fig 2. $D M$ lab is supported by grants from Indian Council of Medical Research, Department of Science and Technology and Department of Biotechnology, Govt of India. SL is the recipient of the Senior Research Fellowship from Council for Scientific and Industrial Research, New Delhi, India. The manuscript bears the NIRRH number IR/731/01-2019.

\section{References}

ALTMÄE S, KOEL M, VÕSA U, ADLER P, SUHORUTŠENKO M, LAISK-PODAR T, KUKUSHKINA V, SAARE M, VELTHUT-MEIKAS A, KRJUTŠKOV K, AGHAJANOVA L, LALITKUMAR PG, GEMZELL-DANIELSSON K, GIUDICE L, SIMÓN C, SALUMETS A (2017). Meta-signature of human endometrial receptivity: a meta-analysis and validation study of transcriptomic biomarkers. Sci Rep 7:10077.

AKBAS EG, SONG J, TAYLOR HS (2004). A HOXA10 estrogen response element (ERE) is differentially regulated by 17 beta-estradiol and diethylstilbestrol (DES). $J$ Mol Biol 340: 1013-1023.

APLIN JD, RUANE PT (2017). Embryo-epithelium interactions during implantation at a glance. J Cell Sci 130: 15-22.

ASHARY N, TIWARI A, MODI D (2018). Embryo Implantation: War in times of love. Endocrinology 159: 1188-1198.

BAGOT CN, KLIMAN HJ, TAYLOR HS (2001). Maternal Hoxa10 is required for pinopod formation in the development of mouse uterine receptivity to embryo implantation. Dev Dyn 222: 538-544.

BAJPAI AK, DAVULURI S, CHANDRASHEKAR DS, ILAKYA S, DINAKARAN M, ACHARYA KK (2012). Mgex-Udb: A mammalian uterus database for expressionbased cataloguing of genes across conditions, including endometriosis and cervical cancer. PLoS One 7: e36776.

BENSON G V., HYUNJUNG LIM, PARIABC, SATOKATA I, DEY SK, MAAS RL (1996). Mechanisms of reduced fertility in Hoxa-10 mutant mice: uterine homeosis and loss of maternal Hoxa-10 expression. Development 122: 2687-2696.

BHUSANE K, BHUTADA S, CHAUDHARI U, SAVARDEKAR L, KATKAM R, SACHDEVA G (2016). Secrets of Endometrial Receptivity: Some Are Hidden in Uterine Secretome. Am J Reprod Immunol 75: 226-236.

CHAVAN AR, GRIFFITH OW, WAGNER GP (2017). The inflammation paradox in the evolution of mammalian pregnancy: turning a foe into a friend. Curr Opin Genet Dev 47: 24-32.

CHENG W, LIU J, YOSHIDA H, ROSEN D, NAORA H (2005). Lineage infidelity of epithelial ovarian cancers is controlled by HOX genes that specify regional identity in the reproductive tract. Nat Med 11:531-537

CHENG Z, ZHU Y, SU D, WANG J, CHENG L, CHEN B, WEI Z, ZHOU P, WANG B MA X, CAO Y (2011). A novel mutation of HOXA10 in a Chinese woman with a Mllerian duct anomaly. Hum Reprod 26: 3197-3201.

DAMSKY CH, LIBRACH C, KEE-HAKL, MARILYN F, MCMASTER M, MARY J, ZHOU Y, LOGAN S, FISHER SJ (1994). Integrin switching regulates normal trophoblast invasion. Development 120: 3657-3666.

DIMITRIADIS E, MENKHORST E, SALAMONSEN LA, PAIVA P (2010). Review: 
LIF and IL11 in trophoblast-endometrial interactions during the establishment of pregnancy. Placenta 31: S99-S104.

DU H, TAYLOR HS (2015). The role of Hox genes in female reproductive tract development, adult function, and fertility. Cold Spring Harb Perspect Med 6: a023002.

EKICI AB, STRISSEL PL, OPPELT PG, RENNER SP, BRUCKER S, BECKMANN MW, STRICK R (2013). HOXA10 and HOXA13 sequence variations in human female genital malformations including congenital absence of the uterus and vagina. Gene 518: 267-72

EVANS J, SALAMONSEN LA, WINSHIP A, MENKHORST E, NIE G, GARGETT CE, DIMITRIADIS E (2016). Fertile ground: Human endometrial programming and lessons in health and disease. Nat Rev Endocrinol 11: 654-667

GAO F, BIAN F, MA X, KALINICHENKO V, DAS SK (2015). Control of regional decidualization in implantation: Role of FoxM1 downstream of Hoxa10 and cyclin D3. Sci Rep 5: 13863.

GELLERSEN B, BROSENS JJ (2014). Cyclic decidualization of the human endometrium in reproductive health and failure. Endocr Rev 35: 851-905.

GODBOLE G, MODI D (2010). Regulation of decidualization, interleukin-11 and interleukin- 15 by homeobox A 10 in endometrial stromal cells. J Reprod Immunol 85: $130-139$.

GODBOLE G, SUMAN P, GUPTASK, MODI D (2011). Decidualized endometrial stromal cell derived factors promote trophoblast invasion. Fertil Steril 95: 1278-1283.

GODBOLE G, SUMANP, MALIKA, GALVANKARM, JOSHIN, FAZLEABASA, GUPTA SK, MODI D (2017). Decrease in Expression of HOXA10 in the Decidua After Embryo Implantation Promotes Trophoblast Invasion. Endocrinol. 158: 2618-2633.

GODBOLE G, MODI D, PURI C (2007). Regulation of homeobox A10 expression in the primate endometrium by progesterone and embryonic stimuli. Reprod. 134: 513-523

GUI Y, ZHANG J, YUAN L, LESSEY BA (1999). Regulation of HOXA-10 and its expression in normal and abnormal endometrium. Mol Hum Reprod 5: 866-873.

HIROTA Y, TRANGUCH S, DAIKOKU T, HASEGAWA A, OSUGA Y, TAKETANI Y, DEY SK (2008). Deficiency of Immunophilin FKBP52 Promotes Endometriosis. Am J Pathol 173: 1747-1757.

JIANG R, DING L, ZHOU J, HUANG C, ZHANG Q, JIANG Y, LIU J, YAN Q, ZHEN X, SUN J, YAN G, SUN H (2017). Enhanced HOXA10 sumoylation inhibits embryo implantation in women with recurrent implantation failure. Cell Death Discov 3: 17057.

KIM JJ, TAYLOR HS, LU Z, LADHANI O, HASTINGS JM, JACKSON KS, WU Y, GUO SW, FAZLEABAS AT (2007). Altered expression of HOXA10 in endometriosis: Potential role in decidualization. Mol Hum Reprod 13: 323-332.

KURIAN NK, MODI D (2018). Extracellular vesicle mediated embryo-endometrial cross talk during implantation and in pregnancy. J Assist Reprod Genet: 1-10.

LAHERI S, ASHARY N, BHATT P, MODI D (2018). Oviductal glycoprotein 1 (OVGP1) is expressed by endometrial epithelium that regulates receptivity and trophoblast adhesion. J Assist Reprod Genet 35: 1419-1429.

LAHERI S, MODI D, BHATT P (2017). Extra-oviductal expression of oviductal glycoprotein 1 in mouse: Detection in testis, epididymis and ovary. J Biosci 42: 69-80.

LIATSIKOS SA, GRIMBIZIS GF, GEORGIOU I, PAPADOPOULOS N, LAZAROS L, BONTIS JN, TARLATZIS BC (2010). HOX A10 and HOX A11 mutation scan in congenital malformations of the female genital tract. Reprod Biomed Online 21: 126-132.

LIM H, MA L, MA WG, MAAS RL, DEY SK (1999). Hoxa-10 regulates uterine stromal cell responsiveness to progesterone during implantation and decidualization in the mouse. Mol Endocrinol 13: 1005-1017.

LU Z, HARDT J, KIM JJ (2008). Global analysis of genes regulated by HOXA10 in decidualization reveals a role in cell proliferation. Mol Hum Reprod 14: 357-66.

LUCASES, VRLJICAKP, DINIZ-DA-COSTAMM, BRIGHTON PJ, KONG CS, LIPECKI J, FISHWICK K, MUTER J, OTT S, BROSENS JJ, BROSENS JJ (2018). Reconstruction of the Decidual Pathways in Human Endometrial Cells Using Single-Cell RNA-Seq. bioRxiv. DOI: http://dx.doi.org/10.1101/368829

MACKLON NS, BROSENS JJ (2014). The Human Endometrium as a Sensor of Embryo Quality. Biol Reprod 91: 1-8.

MANGALE S, MODI D, REDDY KVR (2008). Identification of genes regulated by an interaction between avb3 integrin and vitronectin in murine decidua. Reprod Fertil Dev 20: 311-319.

MODID, BHARTIYAD, PURIC (2006). Developmental expression and cellular distribu- tion of Müllerian inhibiting substance in the primate ovary. Reprod. 132: 443-453.

MODIDN, BHARTIYAP (2015). Physiology of embryo-endometrial cross talk. Biomed Res J 2: 83-104

MODI D, GODBOLE G (2009). HOXA10 signals on the highway through pregnancy. $J$ Reprod Immunol 83: 72-78.

MODI D, GODBOLE G, SUMAN P, GUPTA SK (2012). Endometrial biology during trophoblast invasion. Front Biosci (Schol Ed) 4: 1151-1171.

NIMBKAR-JOSHI S, KATKAM RR, CHAUDHARI UK, JACOB S, MANJRAMKAR DD, METKARI SM, HINDUJA I, MANGOLI V, DESAI S, KHOLKUTE SD, PUR CP (2012). Endometrial epithelial cell modifications in response to embryonic signals in bonnet monkeys (Macaca radiata). Histochem Cell Biol 138: 289-304.

OKADAH, TSUZUKIT, MURATAH(2018). Decidualization of the human endometrium. Reprod Med Biol 17: 220-227.

PADMANABHAN R, LALORAYA M (2016). Estrogen-Initiated Protein Interactomes During Embryo Implantation. Am J Reprod Immunol 75: 256-262.

PARKER HJ, BRONNER ME, KRUMLAUF R (2016). The vertebrate Hox gene regulatory network for hindbrain segmentation: Evolution and diversification. BioEssays 38: 526-538.

PATIL VS, SACHDEVA G, MODI DN, KATKAM RR, MANJRAMKAR DD, HINDUJA I, PURI CP (2005). Rab coupling protein (RCP): A novel target of progesterone action in primate endometrium. J Mol Endocrinol 35: 357-372.

ROSARIO GX, D'SOUZASJ, MANJRAMKARDD, PARMAR V, PURICP, SACHDEVA $G$ (2008). Endometrial modifications during early pregnancy in bonnet monkeys (Macaca radiata). Reprod Fertil Dev 20: 281-94.

ROSARIO GX, KATKAM RR, NIMBKAR-JOSHI S, MODI D, MANJRAMKAR DD, HINDUJAI, ZAVERIK, PURI CP, SACHDEVAG (2009). Expression of Endometria Protein Kinase A During Early Pregnancy in Bonnet Monkeys (Macaca radiata). Biol Reprod 81: 1172-1181.

ROSARIO GX, MODI D, SACHDEVA G, MANJRAMKAR DD, PURI CP (2005a). Morphological events in the primate endometrium in the presence of a preimplantation embryo, detected by the serum preimplantation factor bioassay. Hum Reprod 20: 61-71.

ROSARIO GX, SACHDEVA G, MANJRAMKAR DD, MODI D, MEHERJI PK, PUR CP (2005b). Endometrial expression of immunomodulatory cytokines and their regulators during early pregnancy in bonnet monkeys (Macaca radiata). Hum Reprod 20: 3039-3046.

ROSARIO GX, SACHDEVA G, MANJRAMKAR DD, PURI CP (2005c). Enhanced expressions of endometrial tumour necrosis factor alpha and its receptors during early pregnancy in bonnet monkeys. Cytokine 31: 459-464.

RYTKÖNEN K, ERKENBRACK E, POUTANEN M, ELO L, PAVLICEV M, WAGNER G (2018). Decidualization of Human Endometrial Stromal Fibroblasts is a Multiphasic Process Involving Distinct Transcriptional Programs. Reprod Sci p1933719118802056.

SATOKATA I, BENSON G, MAAS R (1995). Sexually dimorphic sterility phenotypes in hoxalo-deficient mice. Nature 374: 460-463.

SHARMAS, GODBOLE G, MODID (2016). Decidual Control of Trophoblast Invasion. Am J Reprod Immunol 75: 341-350.

SUMAN P, GODBOLE G, THAKUR R, MORALES-PRIETO D, MODI D, MARKERT U, GUPTA S (2012). AP-1 transcription factors, mucin-type molecules and mmps regulate the IL-11 mediated invasiveness of JEG-3 and HTR-8/svneo trophoblastic cells. PLoS One 7: e29745.

STRUGM, SUR, YOUNG J, DODDSW, SHAVELLV, DÍAZ-GIMENOP, RUÍZ-ALONSO M, SIMÓN C, LESSEY B, LEACH R, FAZLEABAS A (2016). Intrauterine human chorionic gonadotropin infusion in oocyte donors promotes endometrial synchrony and induction of early decidual markers for stromal survival: a randomized clinical trial. Hum Reprod 31: 1552-1561.

SURYAWANSHI H, MOROZOV P, STRAUS A, SAHASRABUDHE N, MAX KEA GARZIAA, KUSTAGI M, TUSCHL T, WILLIAMS Z (2018). A single-cell survey of the human first-trimester placenta and decidua. Sci Adv 4: eaau4788

TAYLOR HS, ARICI A, OLIVE D, IGARASHI P (1998). HOXA10 is expressed in response to sex steroids at the time of implantation in the human endometrium. J Clin Invest 101:1379-1384.

TAYLOR H, VANDEN HEUVEL G, IGARASHI P (1997). A Conserved Hox Axis in the Mouse and Human Female Reproductive System: Late Establishment and PersistentAdult Expression of the Hoxa Cluster Genes. Biol Reprod57:1338-1345. 
VENTO-TORMO R, EFREMOVA M, BOTTING RA, TURCO MY, VENTO-TORMO M, MEYER KB, PARK J-E, STEPHENSON E, POLAŃSKI K, GONCALVES A, et al., (2018). Single-cell reconstruction of the early maternal-fetal interface in humans. Nature 563: 347-353

WHITAKER L, MURRAY A, MATTHEWS R, SHAW G, WILLIAMS A, SAUNDERS P, CRITCHLEY H (2017). Selective progesterone receptor modulator (SPRM) ulipristal acetate (UPA) and its effects on the human endometrium. Hum Reprod 32: 531-543.

XU B, GEERTS D, BU Z, AI J, JIN L, LI Y, ZHANG H, ZHU G (2014). Regulation of endometrial receptivity by the highly expressed HOXA9, HOXA11 and HOXD10
HOX-class homeobox genes. Hum Reprod 29: 781-790.

YAO MWM, LIM H, SCHUST DJ, CHOE SE, FARAGO A, DING Y, MICHAUD S, CHURCHGM, MAASRL(2003). Gene Expression Profiling Reveals ProgesteroneMediated Cell Cycle and Immunoregulatory Roles of Hoxa-10in the Preimplantation Uterus. Mol Endocrinol 17: 610-627.

ZHU L, SUN L, HU Y, JIANG Y, LIU H, SHEN X, JIN X, ZHEN X, SUN H, YAN G (2013). PCAF Impairs Endometrial Receptivity and Embryo Implantation by DownRegulating b3-Integrin Expression via HOXA10 Acetylation. J Clin Endocrinol Metab 98: 4417-4428. 


\section{Further Related Reading, published previously in the Int. J. Dev. Biol.}

Breakthroughs and challenges of modern developmental biology and reproductive medicine

Simon Fishel, Anna-Katerina Hadjantonakis, Berenika Plusa, Laura Rienzi, Yojiro Yamanaka, Aneta Suwi\&nacute;skaand Anna Ajduk Int. J. Dev. Biol. (2019) 63: 77-82

https://doi.org/10.1387/ijdb.180398aa

Early mammalian development: from basic research to the clinic

Aneta Suwi\&nacute;ska and Anna Ajduk

Int. J. Dev. Biol. (2019) 63: 73-75

https://doi.org/10.1387/ijdb.180409as

The mammalian embryo's first agenda: making trophectoderm

Eszter Posfai, Isidora Rovic and Andrea Jurisicova

Int. J. Dev. Biol. (2019) 63: 157-170

https://doi.org/10.1387/ijdb.180404ep

Dipeptidyl peptidase IV reduces trophoblast invasion by inhibiting the activity of MMPs

Youfei Li, Zhen Li and Jian Zhang

Int. J. Dev. Biol. (2014) 58: 349-353

https://doi.org/10.1387/ijdb.140016yl

Cell signaling in trophoblast-uterine communication

Rani Fritz, Chandni Jain and D. Randall Armant

Int. J. Dev. Biol. (2014) 58: 261-271

https://doi.org/10.1387/ijdb.140011da

Immunological determinants of implantation success

Sarah A. Robertson and Lachlan M. Moldenhauer

Int. J. Dev. Biol. (2014) 58: 205-217

https://doi.org/10.1387/ijdb.140096sr

T cell behavior at the maternal-fetal interface

Patrice Nancy and Adrian Erlebacher

Int. J. Dev. Biol. (2014) 58: 189-198

https://doi.org/10.1387/ijdb.140054ae
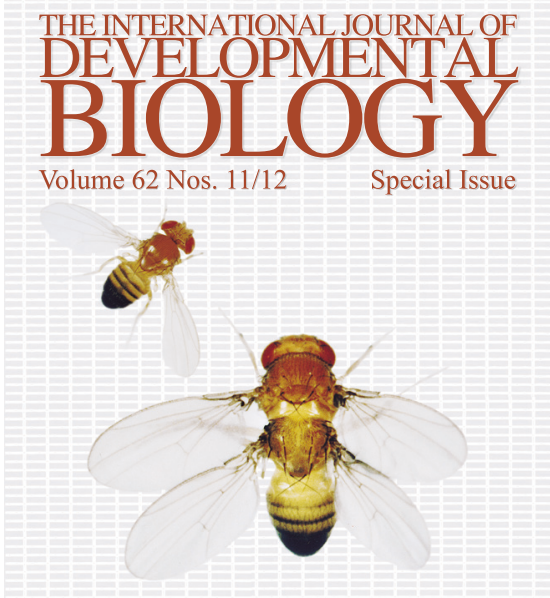

Homeobox Genes
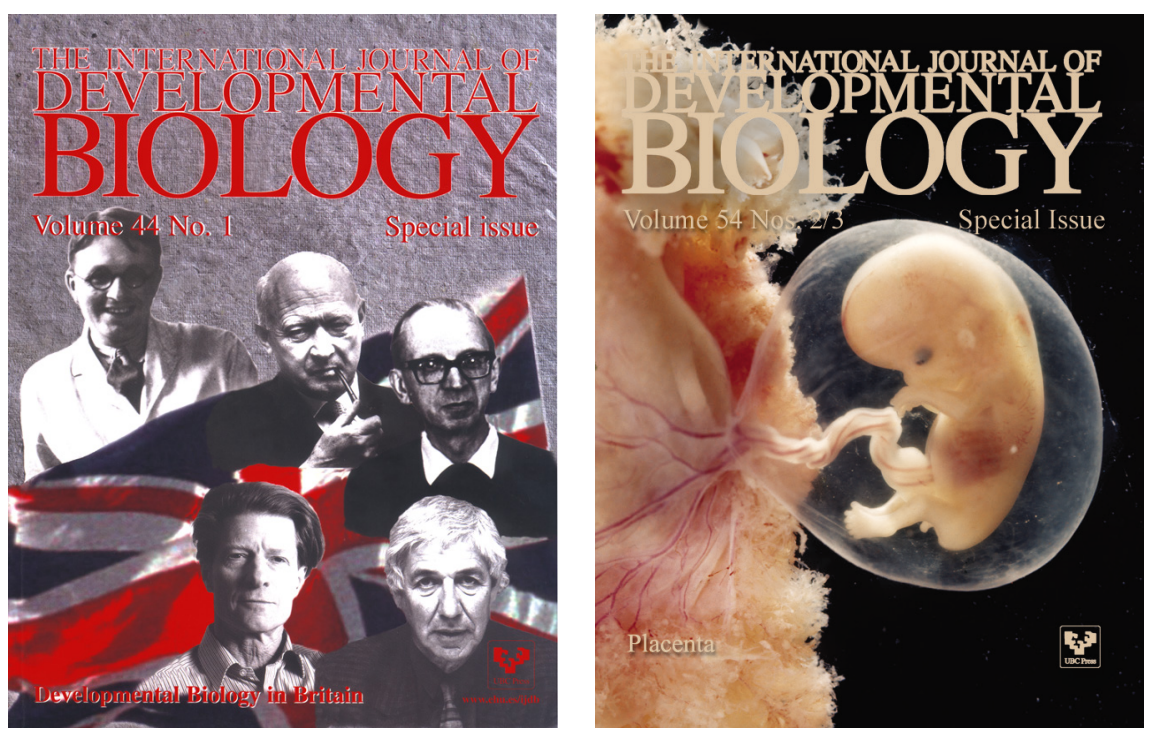